\title{
Progress in the Critical Assessment for a Far-Infrared Space Interferometer with Double Fourier Modulation (FP7-FISICA)
}

\author{
G.Savini*a , P.A.R. Ade ${ }^{\mathrm{b}}$, N.Baccichet ${ }^{\mathrm{a}}$, C.Bracken $^{\mathrm{e}}$, K.Dohlen $^{\mathrm{h}}$, A.Donohoe ${ }^{\mathrm{e}}$, B.Gom ${ }^{\mathrm{f}}$, M.J. \\ Griffin $^{\mathrm{b}}$, W.Holland ${ }^{\mathrm{c}}$, V.Iafolla ${ }^{\mathrm{i}}$, R.J. Ivison ${ }^{\mathrm{c}}$, M.Jones ${ }^{\mathrm{d}}$, R.Juanola-Parramon ${ }^{\mathrm{a}}$, J.Lightfoot ${ }^{\mathrm{c}}$, \\ S.Liu $^{\mathrm{g}}$, A.McMillan ${ }^{\mathrm{d}}$, J.A.Murphy, ${ }^{\mathrm{e}}$, D.Naylor ${ }^{\mathrm{f}}$, C.O’Sullivan $^{\mathrm{e}}$, E.Pascale ${ }^{\mathrm{b}}$, S.Pezzutto ${ }^{\mathrm{g}}$, \\ E.Rakotonimbahy ${ }^{\mathrm{h}}$, D.Schito ${ }^{\mathrm{g}}$, L.Spencerf ${ }^{\mathrm{f}}$ L.Spinoglio ${ }^{\mathrm{g}}$, B.Swinyard ${ }^{\mathrm{a}}$, I.Venendaal ${ }^{\mathrm{f}}$, \\ S.Vives ${ }^{\text {h }}$, D.Walker ${ }^{\mathrm{d}}$, \\ And \\ D.Leisawitz ${ }^{\mathrm{j}}$, S.Shi ${ }^{\mathrm{k}}$, H.Matsuo ${ }^{\mathrm{l}}$ \\ ${ }^{a}$ Dept. of Physics and Astronomy, University College London, Gower Street, London WC1E 6BT, UK; \\ ${ }^{\mathrm{b}}$ School of Physics \& Astronomy, Cardiff University, The Parade, Cardiff CF24 3AA, UK; \\ c UK Astronomy Technology Centre, Science and Technology Facilities Council, Royal Observatory, Blackford \\ Hill, Edinburgh EH9 3HJ, UK; \\ ${ }^{\mathrm{d}}$ Glyndwr University, Optic Technium, St.Asaph, Ffordd William Morgan, St. Asaph Business Park, North \\ Wales LL17 0JD, United Kingdom; \\ ${ }^{\mathrm{e}}$ Dep. of Experimental Physics, National University of Ireland Maynooth, Maynooth, Ireland; \\ ${ }_{\mathrm{f}}^{\mathrm{f}}$ Institute for Space Imaging Science, University of Lethbridge, 4401 University Drive, Lethbridge, AB T1J \\ 1B1, Canada; \\ ${ }^{g}$ INAF-IAPS, Via Fosso del Cavaliere 100, I-00133 Roma, Italy; \\ ${ }^{\mathrm{h}}$ Lab. d'Astrophysique de Marseille, CNRS, Aix-Marseille Univ. France; \\ ${ }^{i}$ Assist in Gravitation and Instrumentation, Via E. Stevenson 3, Monte Porzio Catone, Roma, Italy; \\ ${ }^{j}$ NASA Goddard Space Flight Center, 8800 Greenbelt Rd., Greenbelt, MD, USA 20771-2400; \\ ${ }^{k}$ Purple Mountain Observatory and Key Laboratory of Radio Astronomy, Chinese Academy of Sciences, 2 \\ West Beijing Road, Nanjing, Jiangsu 210008, People's Republic of China; \\ ${ }^{1}$ Advanced Technology Center, National Astronomical Observatory of Japan, Japan \\ *corresponding author: Giorgio Savini, g.savini@ucl.ac.uk
}

\section{SHORT ABSTRACT}

The progress and results of the ongoing FP7-FISICA programme to re-asses the scientific goals of a Far-Infrared Space Interfereometer and push the development of some of its key technology elements are reported.

Keywords: far-infrared, spatio-spectral interferometry, FISICA, SPIRIT, FIRI, SPECS, IR detectors, cryogenic optics

\section{INTRODUCTION}

With the end of the Herschel operations era and its data in the process of being fully exploited, the question on the next steps to be taken from the far-infrared instrumentation community in order to ensure scientific products comparable to those currently being achieved at other wavelengths is in the open. A significant portion of the farinfrared scientific community is focusing on achieving a mission which pushes the sensitivity of its detectors to the limit requiring the cooling of all its optical elements [1]. Existing facilities [2] are on the other hand making available instruments with the highest spectral resolution in this wavelength range [3], but all of the mentioned developments are not making progress (in fact they represent a step back) in angular resolution with respect to the Herschel Space Observatory, other than for the fact that smaller wavelength will resolve higher details albeit pointing at different physics potentially in a post-JWST era. In this scenario, technology preparation is underway in a number of laboratories to demonstrate (given the possibility) interferometry in space in order to achieve improvements in angular resolution of an order of magnitude over current capabilities. Increase in the sensitivity to small scale features has now been recognized as a key step to progress in the knowledge of most fields of astrophysics, from proto-planetary discs to a variety of star-formation scenarios, to chemical enrichment in galaxies and the overall structure of AGNs. NASA and ESA had almost a decade ago performed separate studies 
for both a probe [4] and flagship missions [5,6] highlighting a potential way in which such concepts could be developed. In the meanwhile, research groups across three continents have been working to improve our understanding of direct-detection interferometry when combined to spectroscopy in the so-called Double Fourier Modulation technique[7]. The FP7-FISICA programme supported by the European Commission to re-asses the scientific goals of a Far-Infrared Space Interfereometer and push forward some of its key technology elements while identifying a roadmap to achieve the remaining obstacles is now in its final year and here we plan to show how Fourier Transform Spectroscopy has a paramount role in this new challenging field which can deliver the vision for space-based far-IR interferometry by capitalizing on the existing international collaboration.

\section{SCIENTIFIC REQUIREMENTS}

One of the first goals of the FISICA programme was to identify in detail the relevant science cases which would benefit from high-angular resolution observations complemented by spectroscopy. In the first year of this activity a detailed table [8] was compiled with such science cases (both galactic and extra-galactic) which included more importantly the requirements on the datasets for given observations such as the sensitivity, angularresolution, field-of-view and spectral resolution. Interestingly, in most of the cases considered, the capability of increasing the spatial resolution of the observations relaxes the requirements on spectral resolution which is often used in conjunction with simulations to disentangle components in the astrophysical picture which are degenerate due to their proximity (an exception are the very complex observations performed through the disk of the galactic plane where line contributions from the same molecule present in different arms of the spiral are observed, requiring $\mathrm{R}>3 \cdot 10^{4}$ in order to identify their exact origin).

The reduced requirements on spectral resolution as well as the interest of most cases in the continuum, promote Fourier Transform Spectroscopy as the technique of choice in regards to spectroscopy. Due to the substantial requirements on sensitivity given the small angular nature of the source, FTS instruments can avail themselves of sensitive antenna-coupled detectors such as TESs (Transition Edge Superconducting detectors) or KIDs (Kinetic Inductance Detectors) with NEPs (Noise Equivalent Power) of less than $10^{-18} \mathrm{WHz}^{-1 / 2}$.

In order to assess the implications of many of the instrument parameters involved with such a complex instrument, a sensitivity model was setup linking all the satellite and instrument parameters to its performance. At the same time a preliminary choice of a baseline instrument/satellite design was performed to allow a set of parameters to be defined with the idea of revisiting this design after performance derivatives are determined and specific dependence of given parameters are better understood.

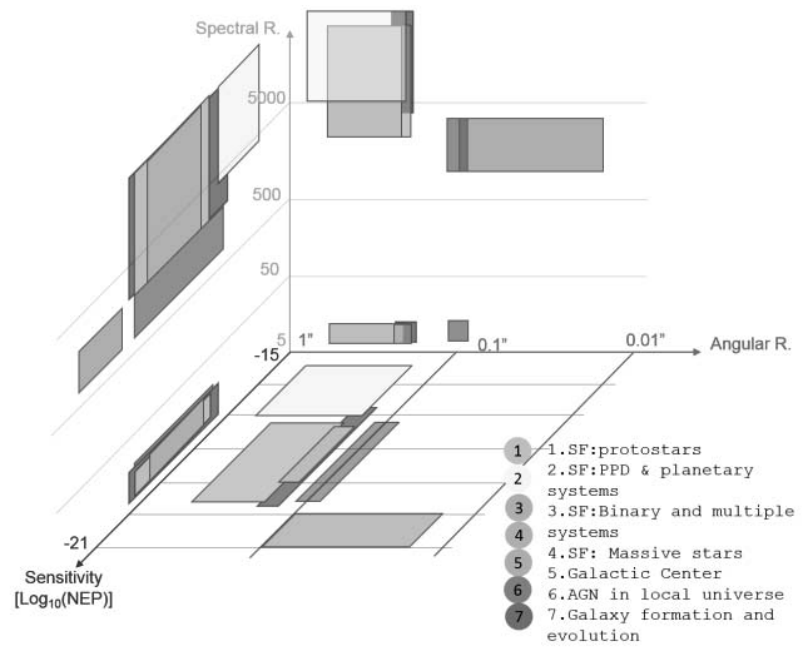

Figure 1. Phase space of required performance for different science cases. These regions of "performance requirement" are indicative of the necessary capabilities (a more numerical full table is contained in a FISICA deliverable available online [8]). A first glance to the plane-projections of the relevant science cases, shows that most cases have a need to produce medium-resolution spectroscopy without forsaking the information on the continuum. Another point is that an increase in angular resolution is accompanied in most cases with an increase in sensitivity albeit this is science case dependent.

A straw-man design of such a mission was devised which was not too distant from the proposed concepts $[4,6]$ in order to build on existing knowledge with a major advantage on a typical feasibility study. Baseline, telescope 
and instrument characteristics were initially chosen led by the science requirements only and then adapted to be self-consistent (meaning that no initial limitation was imposed on baseline size or telescope mass usually constrained by the space vector fairing). While this is not optimal in terms of identifying the readiness of such a mission, it does however focus on the achievability of the science with an "all-out" concept also allowing a subsequent analysis of the trade-offs that occur and the science which is forsaken in the name of feasibility.

The baseline in question was considered to be an $80 \mathrm{~m}$ max baseline with two $2 \mathrm{~m}$ telescopes. Intermediate optics are identified in the sensitivity model in numbers and emissivity as the model does not factor in details of the instrument systematics but rather its general performance. Given the combined requirements identified in the various science cases (Fig.1) the spectral modulation of choice capable of measuring the continuum as well as molecular lines with spectral resolution of the order of a few thousand is identified in FT spectroscopy. The latter lends itself well when combined with direct interferometry to achieve high angular resolution.

\section{DOUBLE-FOURIER MODULATION AND ITS TECHNOLOGY}

The optical path modulating element conceived in the instrument strawman design is based on a pivoting design not dissimilar to an existing design [9] with a difference borrowed from the ESA-CDF design [5] which exploits the definition of the four spectral channels $(25-50-100-200-400 \mu \mathrm{m})$ to have Nyquist frequencies at multiples of that of the longest wavelength channel. This can then be achieved with a single modulating element which introduces path delays which are multiples of that of the shortest wavelength. A double-tower of roof mirrors can be used to this effect with each tower (one for each optical telescope) consisting of a fixed set of roof-mirrors and dichroics (where each channel exists the delay line) and the pivoting element (with the facing roof-tops) modulating the optical path difference with the same factor 2 gain of [9] due to it acting as positive/negative for the two optical ports respectively.

Besides the definition and the expected performance of such an instrument concept, a substantial portion of the FISICA activity is based on the advancement of some of the necessary technological elements to achieve it in the range of wavelengths of interest. An existing DFM testbed which was used in [10] to prove that DFM could be achieved at sub-mm wavelengths is being upgraded to work in the range of wavelengths relevant to a future space instrument $(25-400 \mu \mathrm{m})$. The wavelength range of the latter are identified by the interval between the future JWST observatory on one side $(28.5 \mu \mathrm{m})$ and the furthest-reaching bands of the ALMA interferometer in Chile allowed by atmospheric transmission $(\sim 300 \mu \mathrm{m})$ with small amounts of overlap foreseen for cross-calibration.

A first activity which has seen its first results is the build of two broadband beam-dividers working respectively in the range $(20-50 \mu \mathrm{m}$ and $25-100 \mu \mathrm{m})$ with average power recombined of $(\mathrm{T}+\mathrm{R})-(|\mathrm{T}-\mathrm{R}|)=0.9$ and 0.7 in the two respective cases. While their use in the DFM testbed in Cardiff University will also provide validation for the phase behavior of the beam-combiners, the $\mathrm{R}$ and $\mathrm{T}$ measurements and the close match with the model predictions suggest that the beam combiners for such an instrument are achievable with an existing high TRL technology and their performance is simply a matter of parameter tuning through subsequent iterations. Beam combination at wavelengths higher than 100mm have already been proven and flown [11] and will likely require minor improvements.

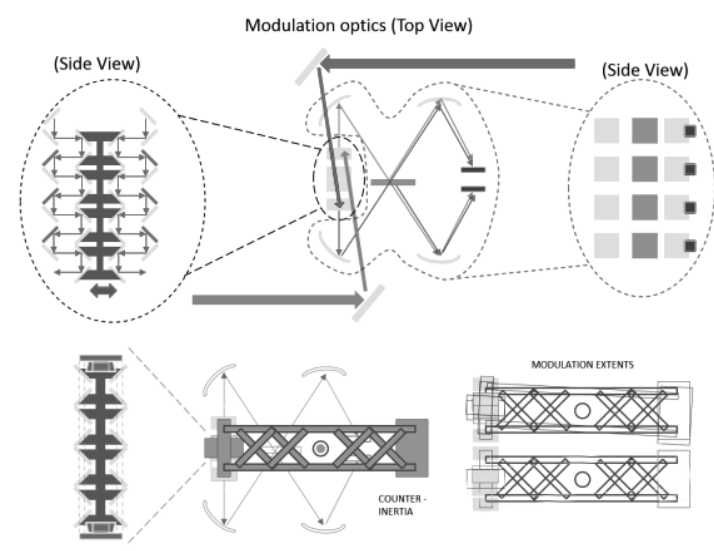

Figure 2. Proposed scheme of optical modulation for the spectral modulation element of the strawman design. The two beams entering the hub are then directed on the opposite arms of the modulating element. This arrangement does not take into account necessary requirements for equal focal length at the detector planes. But is indicative of a potential spatial arrangement of such a modulating element.

Parallel activities are being undertaken within this programme on a cryogenic delay line for detailed metrology 
characterization (see Ian Veneendal et al.), a complex calibration scene to achieve periodic testing of spectral as well as spatial features in a DFM testbed (see Baccichet et al.), work on an end-to-end simulator aimed at predicting the impact of a variety of physical systematics on the performance of a space mission employing DFM (see J.Lightfoot et al. and R.Juanola-Parramon et al.) as well as ongoing work on two different test-beds (see R.Juanola-Parramon et al.).

\section{FUTURE WORK}

With a little more than a year to go at the time of writing, many activities are working to make progress on various technology fronts related to both instrument and satellite. Here we hope to have highlighted that Fourier Spectroscopy through DFM has a key role to play in a potential future instrument for High Angular resolution in the Far Infrared. In the coming months we hope to be able to make progress in the maturity of this technique at wavelengths shorter than those achieved in [10] in two independent test-beds and provide, through detailed simulations of the physics involved, a map and timeline of the relevant technologies' status and the relevant required developments.

\section{REFERENCES}

1. T.Onaka and T.Nakagawa, "SPICA: A $3.5 \mathrm{~m}$ space infrared telescope for mid-and far-infrared astronomy", Advances in Space Research 36, 6, 1123-1127 (2005).

2. P.Temi et al. "The SOFIA Observatory at the Start of Routine Science Operations: Mission Capabilities and Performance", The Astrophysical Journal Supplement, 212, 2, p.13 (2014)

3. S. Heyminck et al. "GREAT: the SOFIA high-frequency heterodyne instrument", Astronomy \& Astrophysics, 542, L1 (2012).

4. Leisawitz, D., Baker, C., Barger, A., et al., "The space infrared interferometric telescope (SPIRIT): Highresolution imaging and spectroscopy in the far-infrared," Advances in Space Research 40, 689703 (2007).

5. ESA Concurrent Design Facility, FIRI CDF Study Report, CDF-49(A), (2006). http://sci.esa.int/futuremissions-office/40738-firi-cdf-study-report/

6. D.Leisawitz, et al., "SPECS: the kilometer-baseline far-IR interferometer in NASA's space science roadmap", Proceedings of the SPIE, 5487, 1527-1537 (2004).

7. Mariotti, J. and Ridgway, S. T., "Double Fourier spatio-spectral interferometry - Combining high spectral and high spatial resolution in the near infrared," Astron. Astrophys. 195, 350-363 (Apr. 1988).

8. L.Spinoglio et al. in preparation

9. B.M.Swinyard et al. "Imaging FTS for Herschel SPIRE", Proceedings of the SPIE, 4850, 698-709, (2003).

10. Grainger, W. F., Juanola-Parramon, R., Ade, P. A. R., et al., "Demonstration of spectral and spatial interferometry at THz frequencies," Appl. Opt. 51, 2202-2211 (Apr 2012)

11. D.Naylor et al. "In-orbit performance of the Herschel/SPIRE imaging Fourier transform spectrometer: lessons learned", Proceedings of the SPIE, 9143, id. 91432D p. 15 (2014). 